\title{
A Comparison Study for Investigation of Diffracted Waves between Parallel Edges and Edges with Arbitrary Angle
}

\author{
Jiro Iwashige, ${ }^{1}$ Leonard Barolli, ${ }^{1}$ Motohiko Iwaida, ${ }^{2}$ and Saki Kameyama ${ }^{2}$ \\ ${ }^{1}$ Department of Information and Communication Engineering, Fukuoka Institute of Technology (FIT), 3-30-1 Wajiro-Higashi, \\ Higashi-Ku, Fukuoka 811-0295, Japan \\ ${ }^{2}$ Graduate School of Engineering, Fukuoka Institute of Technology (FIT), 3-30-1 Wajiro-Higashi, Higashi-Ku, Fukuoka 811-0295, Japan \\ Correspondence should be addressed to Leonard Barolli; barolli@fit.ac.jp
}

Received 4 July 2013; Accepted 1 October 2013

Academic Editor: David Taniar

Copyright (C) 2015 Jiro Iwashige et al. This is an open access article distributed under the Creative Commons Attribution License, which permits unrestricted use, distribution, and reproduction in any medium, provided the original work is properly cited.

\begin{abstract}
Estimation of the scattering of electromagnetic waves by buildings and other obstacles is very important for wireless communications. In our previous work, we have examined the diffracted fields by two horizontal edges which make an arbitrary angle. In this paper, we compare the diffracted fields between parallel edges and edges with arbitrary angle. The numerical calculation results show that when the angle between two edges increases, relatively strong orthogonal polarized wave components appear, but the principal components are almost the same as the incident wave.
\end{abstract}

\section{Introduction}

Electromagnetic waves can be scattered by edges of the buildings in urban areas or edges of the mountain (ridges) for long communication. Thus, the communication quality deteriorates. Therefore, it is important to estimate propagation path from the viewpoint of the effective utilization of the radio wave resources. However, the prediction of the propagation path is not easy.

Until now, there are many research works dealing with estimation of the radio wave propagation path in the urban areas $[1,2]$. These papers mainly analyzed and examined the propagation path of radio waves when in the urban area there are many buildings. For the case when the waves exceed building roof, Zhang analyzed propagating radio wave over a large number of buildings [3].

In our previous works, we have examined the diffracted fields by two horizontal edges which make an arbitrary angle $[4,5]$. But it is almost impossible to find diffraction points on these edges analytically [6], so we used a method which can find the diffraction points by repetition process [5]. However, it should be noted that edges are not always horizontal. Thus, it is necessary to examine the diffracted field by edges which change in both horizontal and vertical directions [7]. We also discussed the fields diffracted by wedges and showed that wedge shapes, materials, and incident polarization do not have significant effect on diffracted fields except near the wedge surfaces [8].

In this paper, first we survey the double diffraction by two edges which make an arbitrary angle. Then, we refer to coordinate transformation between a total coordinate system and an edge-fixed one and explain the method for finding the diffraction points on two edges with an arbitrary angle which change in both horizontal and vertical directions. For the analysis of diffracted fields, we used GTD (geometrical theory of diffraction) $[9,10]$. We make the analysis by including the slope diffraction considering Holm's method $[11,12]$. The numerical calculations are carried out on perfect conductor. The results show that when the angle between two edges increases, relatively strong orthogonal polarized wave components appear, but the principal components are the same as the incident wave.

This paper is organized as follows. In Section 2, we present diffraction by two edges containing coordinate transformation and the method of finding the diffraction points and introduce the diffraction by two edges. In Section 3, we present the numerical examples. Finally, some conclusions are given in Section 4. 


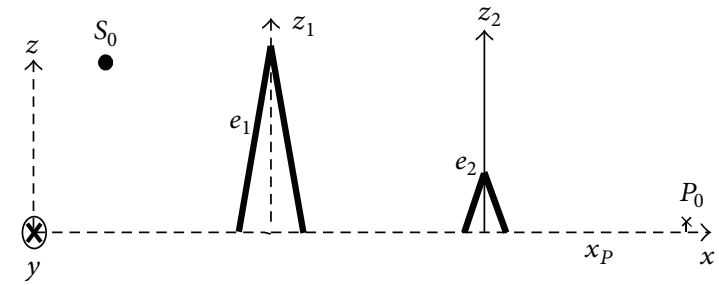

(a) Front view of two wedges

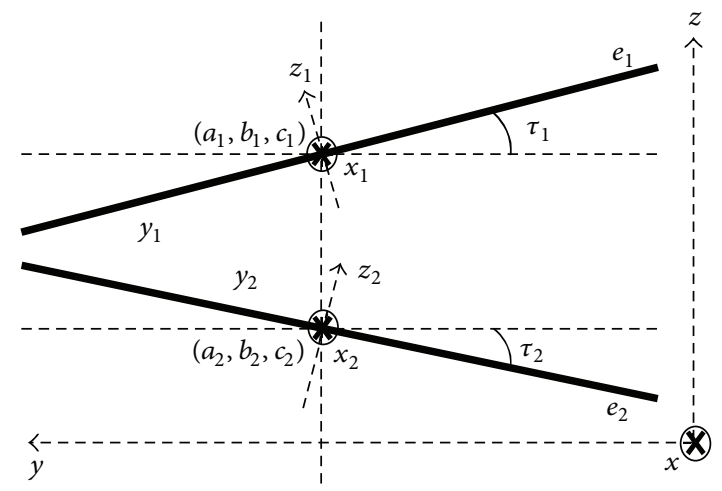

(c) Side view of two edges $e_{1}$ and $e_{2}$

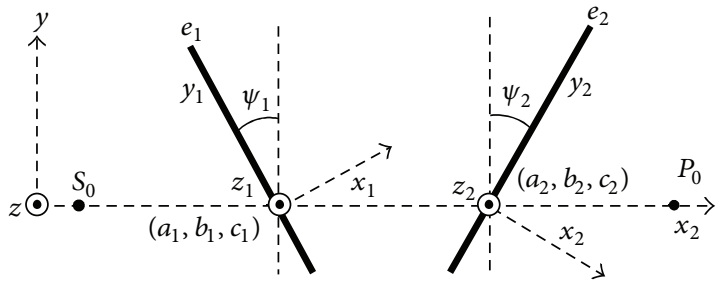

(b) Top view of two edges $e_{1}$ and $e_{2}$

Figure 1: Diffraction by two edges.

\section{Diffraction by Two Edges}

2.1. Aspect of Double Diffraction. In Figure 1(d), the aspect of double diffraction by edges 1 and 2 is shown. We express edge $i(i=1,2)$ by $e_{i}$, which makes an arbitrary angle in horizontal and vertical planes. The wave from a source $S_{0}\left(x_{S}, y_{S}, z_{S}\right)$ is diffracted twice, at point $Q_{1}$ on $e_{1}$ and at point $Q_{2}$ on $e_{2}$, and it reaches an observation point $P_{0}\left(x_{P}, y_{P}, z_{P}\right)$. We use three coordinate systems \#0,\#1 and \#2, and the origins of \#1 and $\# 2$ are, respectively, at $\left(a_{1}, b_{1}, c_{1}\right)$ and $\left(a_{2}, b_{2}, c_{2}\right)$ of coordinate system \#0. The edges $e_{1}$ and $e_{2}$ agree with $y_{1}$-axsis and $y_{2}$ axsis of coordinate systems \#1 and \#2. It should be noted that the subscript $i$ in $(x, y, z)_{i}$ means the coordinate system $\# i$, but the subscript 0 of \#0 is omitted. Figure 1(a) shows the front view of two wages. Figures 1(b) and 1(c) show the top view and side view of Figure 1(d). The wedges are supposed to rotated by angle $\psi_{i}(i=1,2)$ counterclockwise in a horizontal plane as shown in Figure 1(b) and inclined by angle $\tau_{i}$ in a vertical plane in Figure 1(c) (in Figure 1, $\psi_{2}$ and $\tau_{2}$ are drawn as $\psi_{2}<0$ and $\left.\tau_{2}<0\right)$.

2.2. Coordinate Transformation. It is difficult to analyze the diffracted field by edges with an arbitrary angle. But the analysis becomes easier by using coordinate transformation as described below.

Let us consider Figure 1 to explain the coordinate transformation. The origin of coordinate system $\# i(i=1,2)$ is put at $\left(a_{i}, b_{i}, c_{i}\right)$ of coordinate system $\# 0$, and $\# i$ is supposed to be rotated by $\psi_{i}$ counterclockwise around $z_{i}$-axis ( $\# i z$-axis) as shown in Figure 1(b). Furthermore, the $z_{i}$-axis is inclined by $\tau_{i}$ around $x_{i}$-axis (Figure $\left.1(\mathrm{c})\right)$. A point $\left(x_{i}, y_{i}, z_{i}\right)_{i}$ on $\# i$ is obtained from a point $(x, y, z)$ on $\# 0$ by the following equation:

$$
\begin{gathered}
\left(\begin{array}{c}
x_{i} \\
y_{i} \\
z_{i}
\end{array}\right)=T_{i 0}\left(\begin{array}{c}
x-a_{i} \\
y-b_{i} \\
z-c_{i}
\end{array}\right), \\
T_{i 0}=\left(\begin{array}{ccc}
1 & 0 & 0 \\
0 & \cos \tau_{i} & \sin \tau_{i} \\
0 & -\sin \tau_{i} & \cos \tau_{i}
\end{array}\right)\left(\begin{array}{ccc}
\cos \psi_{i} & \sin \psi_{i} & 0 \\
-\sin \psi_{i} & \cos \psi_{i} & 0 \\
0 & 0 & 1
\end{array}\right) .
\end{gathered}
$$

On the other hand, a point $(x, y, z)$ on $\# 0$ is obtained from a point $\left(x_{i}, y_{i}, z_{i}\right)_{i}$ on $\# i$ as follows:

$$
\begin{gathered}
\left(\begin{array}{l}
x \\
y \\
z
\end{array}\right)=T_{0 i}\left(\begin{array}{l}
x_{i} \\
y_{i} \\
z_{i}
\end{array}\right)+\left(\begin{array}{l}
a_{i} \\
b_{i} \\
c_{i}
\end{array}\right), \\
T_{0 i}=\left(\begin{array}{ccc}
\cos \psi_{i} & -\sin \psi_{i} & 0 \\
\sin \psi_{i} & \cos \psi_{i} & 0 \\
0 & 0 & 1
\end{array}\right)\left(\begin{array}{ccc}
1 & 0 & 0 \\
0 & \cos \tau_{i} & -\sin \tau_{i} \\
0 & \sin \tau_{i} & \cos \tau_{i}
\end{array}\right) .
\end{gathered}
$$

In addition, the relationship between a vector $\mathbf{A}=\widehat{\mathbf{x}} A_{x}+$ $\widehat{\mathbf{y}} A_{y}+\widehat{\mathbf{z}} A_{z}$ on $\# 0$ and a vector $\mathbf{A}_{i}=\widehat{\mathbf{x}}_{i} A_{i x}+\widehat{\mathbf{y}}_{i} A_{i y}+\widehat{\mathbf{z}}_{i} A_{i z}$ on $\# i$ is shown as follows:

$$
\begin{aligned}
& \mathbf{A}_{i}=T_{i 0} \mathbf{A}, \\
& \mathbf{A}=T_{0 i} \mathbf{A}_{i} .
\end{aligned}
$$




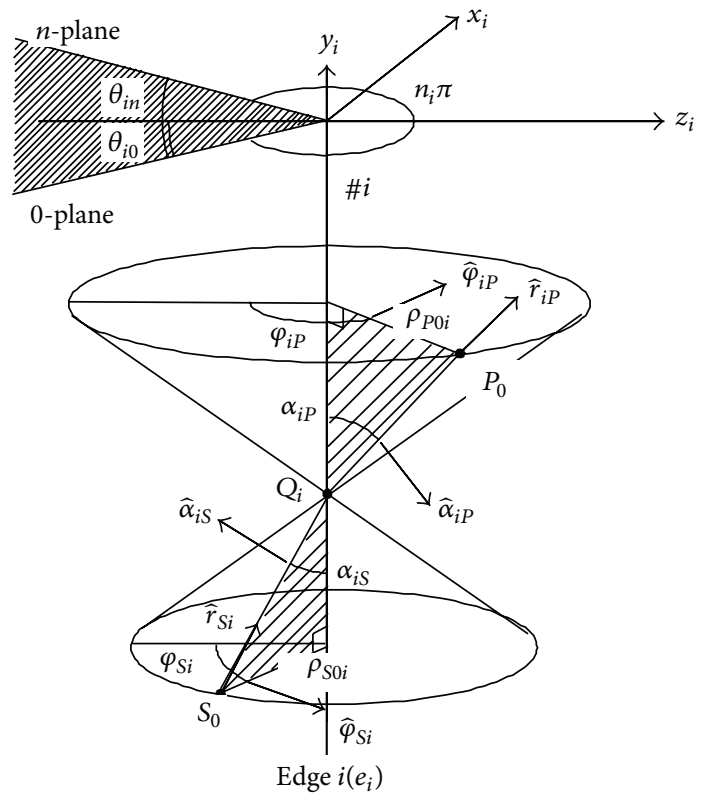

Figure 2: Aspect of diffraction by an edge $e_{i}$.

2.3. Diffracted Field by Edge $e_{i}$. Firstly, we would consider a diffraction by an edge $e_{i}(i=1,2)$ of a wedge $i$. The aspect of diffraction at $Q_{i}$ on an edge $e_{i}$ on the coordinate system $\# i$ is shown in Figure 2. The wedge which constitutes $e_{i}$ has the external angle $n_{i} \pi$ and it consists of 0-plane and $n$-plane which are in the angle of $\theta_{i 0}, \theta_{i n}$ from the $z_{i}\left(z_{i}<0\right)$ axis. The source point $S_{0}$, the observation point $P_{0}$, and a unit vector of the current at $S_{0}$ are expressed as $S_{0}\left(x_{i S}, y_{i S}, z_{i S}\right)_{i}, P_{0}\left(x_{i P}, y_{i P}, z_{i P}\right)_{i}$, and $\hat{\mathbf{i}}_{i}$ of coordinate system $\# i$. The electromagnetic wave emitted from the source $S_{0}$ is diffracted at point $Q_{i}$ on $e_{i}$ and reaches the observation point $P_{0}$. According to GTD, as the angle $\alpha_{i S}$ between the incident path $S_{0} Q_{i}$ and $e_{i}$ ( $y_{i}$ axis) is equal to the angle $\alpha_{i P}$ between the path $Q_{i} P_{0}$ after the diffraction and $e_{i}$ [9], the point $Q_{i}\left(0, y_{\mathrm{Q} i}, 0\right)_{i}$ is easily obtained as follows:

$$
y_{\mathrm{Q} i}=\frac{\rho_{P 0 i} y_{i S}+\rho_{S 0 i} y_{i P}}{\rho_{P 0 i}+\rho_{S 0 i}},
$$

where $\rho_{P 0 i}$ and $\rho_{S 0 i}$ are the distances from the $e_{i}$ to the points $P_{0}$ and $S_{0}$, respectively. Thus, the distances, the angles, the unit vectors $\widehat{\boldsymbol{r}}_{S i}$ and $\widehat{\boldsymbol{\alpha}}_{i S}$, and so on in Figure 2 can be calculated.

Therefore, the single diffracted field $\mathbf{E}_{i}^{d 1}$ at point $Q_{i}$ on the $e_{i}$ is obtained by the following equations [10-12]:

$$
\mathbf{E}_{i}^{d 1}=E_{0} \frac{e^{-j k r_{S i P}}}{r_{S i P}} \sqrt{\frac{r_{S i P}}{r_{S i} r_{i P}}}\left(\widehat{\mathbf{r}}_{S i} \times \widehat{\mathbf{i}}_{i} \times \widehat{\mathbf{r}}_{S i}\right) \cdot \overline{\mathbf{D}}_{S i P},
$$

where $E_{0}$ is a constant and $k$ is the wave number. The $r_{S i}$ and $r_{i P}$ are the distances between $S_{0}$ and $Q_{i}$ and between $Q_{i}$ and $P_{0}$, respectively, and $r_{S i P}=r_{S i}+r_{i P}$. The $\widehat{\mathbf{r}}_{S i}$ and $\widehat{\mathbf{i}}_{i}$ are unit vectors of $r_{S i}$ and the current at the source $S_{0}$. The $\overline{\mathbf{D}}_{S i P}$ is the dyadic diffraction coefficient of the diffracted wave from $S_{0}$ to $Q_{i}$ until it reaches $P_{0}$. The diffracted field $\mathbf{E}_{i}^{d 1}$ in (5) described by $\# i$ is easily transformed to $E^{d 1}$ on $\# 0$ using (3).
2.4. Diffraction Points $Q_{1}$ and $Q_{2}$ on Two Edges. When edges make arbitrary angle in a plane or they are parallel in different heights, the diffraction points can be found easily [5]. But, when they make arbitrary angle in the space, it is impossible to find the diffraction points analytically. So, we use the following method to find the diffraction points [7] as shown in Figure 3.

(1) Let us assume $Q_{1}\left(0, y_{\mathrm{Q} 1}, 0\right)_{1}$, which is the diffraction point on $e_{1}$ as given in preceding subsection, to be a first diffraction point $Q_{1}^{(1)}$ (subscript 1 means diffraction point on $e_{1}$ and superscript (1) means 1st step for finding the last diffraction point).

(2) The temporary diffraction point $Q_{2}^{(1)}$ on the edge $e_{2}$ is given as follows (see Figure 3(a)).

(a) A plane $A_{1}$ can be defined containing the point $Q_{1}^{(1)}$ and $e_{2}$.

(b) $S_{0}$ is rotated around $e_{1}$ with a constant distance of $\rho_{S 01}$ as shown in Figure 2; then $S_{0}$ reaches a point $S_{1}\left(x_{S 12}, y_{S 12}, z_{S 12}\right)_{2}$ on the plane $A_{1}$.

(c) Then using the distance from $S_{1}$ to $e_{2}\left(\rho_{S 12}\right)$ and the distance from $P_{0}$ to $e_{2}\left(\rho_{P 02}\right)$, the first temporary diffraction point $Q_{2}^{(1)}\left(0, y_{\mathrm{Q} 21}, 0\right)_{2}$ on $e_{2}$ is given by

$$
y_{\mathrm{Q} 21}=\frac{\rho_{P 02} y_{S 12}+\rho_{S 12} y_{P 02}}{\rho_{P 02}+\rho_{S 12}} .
$$

(3) The 2nd temporary diffraction point $Q_{1}^{(2)}$ on the edge $e_{1}$ is given as follows.

(a) A plane $A_{2}$ can be constructed using the point $Q_{2}^{(1)}$ and $e_{1}$.

(b) $P_{0}$ is rotated around $C_{P 2}$ with the length $\rho_{P 02}$ shown in Figure 3(a), and then $P_{0}$ reaches a point $P_{2}\left(x_{P 21}, y_{P 21}, z_{P 21}\right)_{1}$ on the plane $A_{2}$ as shown in Figure 3(b).

(c) Using $\rho_{S 01}$ and $\rho_{P 21}$, the 2nd temporary diffraction point $Q_{1}^{(2)}\left(0, y_{\mathrm{Q} 12}, 0\right)_{1}$ on $e_{1}$ is given by

$$
y_{\mathrm{Q} 12}=\frac{\rho_{P 21} y_{S 01}+\rho_{S 01} y_{P 21}}{\rho_{P 21}+\rho_{S 01}} \text {. }
$$

(4) After that, we repeat the processes (2) and (3) until $\Delta y_{\text {Qin }}=\left|y_{\text {Qin }}-y_{\mathrm{Q} i(n-1)}\right|(i=1,2 ; n=2,3, \ldots)$ becomes small enough. Then, we can find the last diffraction points $Q_{i}\left(\simeq Q_{i}^{(n)}\right)$.

2.5. Double Diffracted Fields. The double diffracted field $\mathbf{E}^{d 2}$ by the point $Q_{1}$ on the edge $e_{1}$ and the point $Q_{2}$ on the edge $e_{2}$ is calculated by the following equation considering the slope diffraction [10-12]:

$$
\begin{aligned}
\mathbf{E}^{d 2}= & E_{0} \frac{e^{-j k r_{S 12 P}}}{r_{S 12 P}} \sqrt{\frac{r_{S 12 P}}{r_{S 1} r_{12} r_{2 P}}}\left(\widehat{\mathbf{r}}_{S 1} \times \widehat{\mathbf{i}}_{1} \times \widehat{\mathbf{r}}_{S 1}\right) \\
& \cdot\left(\overline{\mathbf{D}}_{S 12} \cdot \overline{\mathbf{D}}_{12 P}+\frac{j}{k r_{12}} \frac{\partial \overline{\mathbf{D}}_{S 12}}{\partial \varphi_{12}} \cdot \frac{\partial \overline{\mathbf{D}}_{12 P}}{\partial \varphi_{21}}\right) \\
= & \mathbf{E}_{(1)}^{d 2}+\mathbf{E}_{(2)}^{d 2},
\end{aligned}
$$




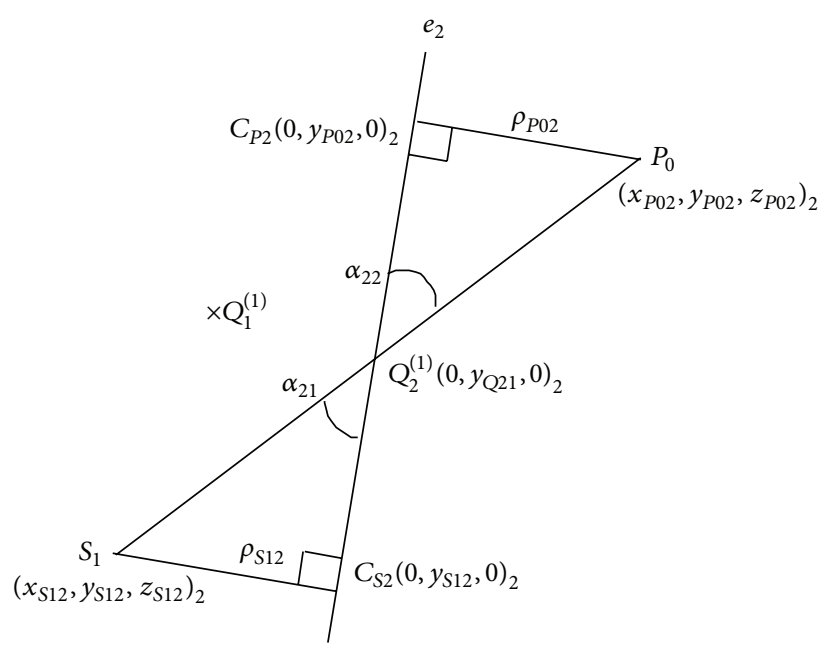

(a) Plane $A_{1}$

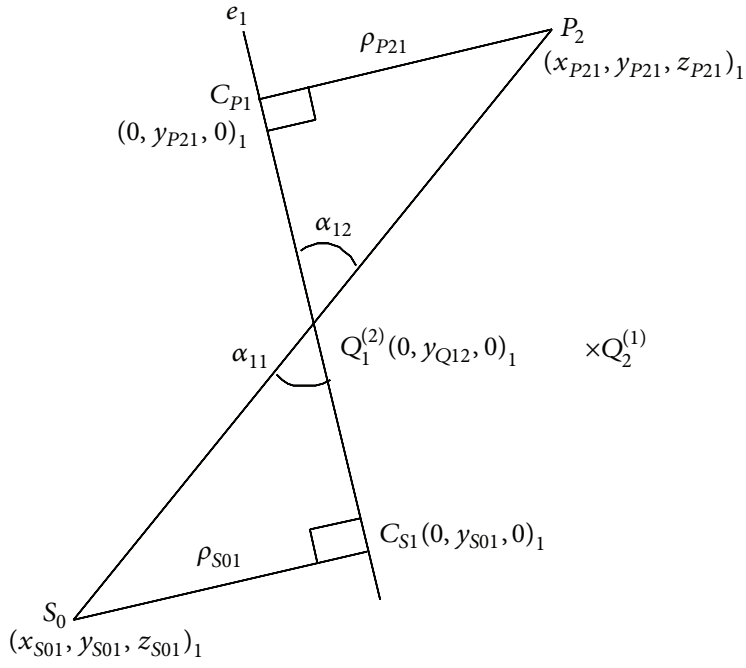

(b) Plane $A_{2}$

Figure 3: Diffraction points on edges $e_{2}$ and $e_{1}$.

where $r_{S 12 P}=r_{S 1}+r_{12}+r_{2 P}$ and $\varphi_{i j}(i, j=1,2)$ is the direction angle at the point $Q_{i}$ toward the point $Q_{j}$ measured from the 0 -plane of the wedge $i$. The first term in the parenthesis of (8) is the first-order diffraction $\left(\mathbf{E}_{(1)}^{d 2}\right)$ and the second term is the second-order diffraction $\left(\mathbf{E}_{(2)}^{d 2}\right.$ : the socalled slope diffraction). When we execute (8), the dyadic diffraction coefficients $\overline{\mathbf{D}}_{S 12}$ and $\overline{\mathbf{D}}_{12 P}$ are calculated on \#1 and \#2, respectively. Then, they are transformed to \#0 by (3), and the double diffracted fields by $e_{1}$ and $e_{2}$ are obtained by performing scalar product in (8).

2.6. Total Field. The direct field $\mathbf{E}^{\mathrm{inc}}$ from a source $S$ to the observation point $P$ is obtained as follows:

$$
\mathbf{E}^{\mathrm{inc}}=E_{0} \frac{e^{-j k r_{S P}}}{r_{S P}}\left(\widehat{\mathbf{r}}_{S P} \times \widehat{\mathbf{i}} \times \widehat{\mathbf{r}}_{S P}\right),
$$

where $r_{S P}$ is the distance between $S$ and $P$, and the $\widehat{\mathbf{r}}_{S P}$ and $\widehat{\mathbf{i}}$ are unit vectors of $r_{S P}$ and the current at $S$ for the coordinate system \#0.

The single diffracted field $\mathbf{E}_{i}^{d 1}(i=1,2)$ by an edge $e_{i}$ and double diffracted field $\mathrm{E}^{d 2}$ by $e_{1}$ and $e_{2}$ can be obtained by (5) and (8). Considering direct field $\mathbf{E}^{\text {inc }}$ from a source $S$ and the field $\mathbf{E}^{\text {ref }}$ reflected by surfaces of the wedges, the total electric field $\mathbf{E}^{t}$ at an observation point $P$ is calculated as the sum of these fields:

$$
\mathbf{E}^{t}=\mathbf{E}^{\mathrm{inc}}+\mathbf{E}^{\mathrm{ref}}+\mathbf{E}_{1}^{d 1}+\mathbf{E}_{2}^{d 1}+\mathbf{E}^{d 2} .
$$

\section{Numerical Examples}

In this section, we present the numerical calculation. The calculations were carried out for the case where the origin of coordinate system \#1 is located at $\left(a_{1}, b_{1}, c_{1}\right)=\left(-50 \mathrm{~m}, 0, h_{1}\right)$ of coordinate system $\# 0$ and for $\# 2$ at $\left(a_{2}, b_{2}, c_{2}\right)=\left(0,0, h_{2}\right)$, where $h_{1}$ and $h_{2}$ are the heights of each origin for the coordinate system $\# 0$ as shown in Figure 1. The angles of edges with $y$-axes are $\psi_{1}, \psi_{2}, \tau_{1}$, and $\tau_{2}$ as shown in Figures $1(\mathrm{~b})$ and $1(\mathrm{c})$, but we do not consider the field diffracted by an edge formed at an intersection of two wedges and the field reflected by the wedge surfaces and the ground. The source $S_{0}$ is at $\left(x_{S}, y_{S}, z_{S}\right)=\left(-100 \mathrm{~m}, 0, h_{S}\right)$, the observation point $P_{0}$ is at $\left(x_{P}, y_{P}, z_{P}\right)=\left(x_{P}, 0,1.5 \mathrm{~m}\right)$, and $E_{0}=1$ in (5), (8), and (9).

When the difference $\left|\psi_{1}-\psi_{2}\right|$ or $\left|\tau_{1}-\tau_{2}\right|$ becomes large, two edges cross each other near to $x$-axis or $z$-axis as shown in Figure 1, and the diffraction points $Q_{1}$ and/or $Q_{2}$ go out the calculable range. So, we firstly checked the limitation of $\psi_{1}$, $\psi_{2}$ and $\tau_{1}, \tau_{2}$ values. We found that we can nearly calculate diffracted fields when $\left|\psi_{1}-\psi_{2}\right|<90^{\circ}$ or $\left|\tau_{1}-\tau_{2}\right|<90^{\circ}$.

In Table 1 the aspect of converging the locations of the diffracted points on two edges $e_{1}$ and $e_{2}$ is shown. Let the heights of the source and the edges be $h_{S}=40 \mathrm{~m}, h_{1}=10 \mathrm{~m}$, and $h_{2}=30 \mathrm{~m}$ and let the angles $\psi_{1}$ and $\psi_{2}$ be $30^{\circ}$ and $-30^{\circ}$, respectively. Table 1 shows the effect of converging locations of $Q_{i}\left(0, y_{Q i}, 0\right)_{i}(i=1,2)$ and the diffraction angles $\alpha_{i}$ at $x_{P}=$ $50 \mathrm{~m}$ and $300 \mathrm{~m}$. In this table, $n$ is the number of repetitions, $\Delta y_{\mathrm{Q} i n}=\left|y_{\mathrm{Q} i n}-y_{\mathrm{Q} i(n-1)}\right|$, and $\Delta \alpha_{i}=\left|\alpha_{i 1}-\alpha_{i 2}\right|$. According to GTD, the angle $\alpha_{i 1}$ between the incident ray and $e_{i}$ is equal to the angle $\alpha_{i 2}$ between $e_{i}$ and the diffracted ray as shown in Figure 2. Table 1(a) is for horizontal edges $\left(\tau_{1}=\tau_{2}=0^{\circ}\right)$ and Table $1(\mathrm{~b})$ is for $\tau_{1}=10^{\circ}$ and $\tau_{2}=-10^{\circ}$. The number of repetitions $n$ has a tendency to increase when $\psi_{i}, \tau_{i}$, and/or $x_{p}$ become large. Although the calculations were carried out for many cases of various angles $\psi_{i}, \tau_{i}$ and various positions of the $\# i$ origins, their results show that the number of repetitions $n$ is relatively small.

In [8], we mentioned that the diffracted fields by wedges are not affected too much by their shapes, material, and incident polarization except near the surfaces of wedges. So, we show the following calculation for the perfectly conducting wedges whose inner angles are zero $\left(\theta_{i 0}=\theta_{\text {in }}=0^{\circ} ; i=1,2\right)$. 
TABLE 1: Relation between $\Delta y_{\mathrm{Qni}}, \Delta \alpha_{i}(i=1,2)$, and $n .\left(h_{1}=10 \mathrm{~m}\right.$, $h_{2}=30 \mathrm{~m}$ and $\psi_{1}=30^{\circ}, \psi_{2}=-30^{\circ}$ ).

$$
\text { (a) } \tau_{1}=\tau_{2}=0^{\circ}
$$

\begin{tabular}{cccccc}
\hline$x_{P}[\mathrm{~m}]$ & $n$ & $\Delta y_{\mathrm{Q} n 1}[\mathrm{~m}]$ & $\Delta y_{\mathrm{Q} n 2}[\mathrm{~m}]$ & $\Delta \alpha_{1}[\mathrm{deg}]$ & $\Delta \alpha_{2}[\mathrm{deg}]$ \\
\hline \multirow{4}{*}{50} & 1 & 1.0 & - & $1.8 \times 10^{-1}$ & 1.2 \\
& 2 & $2.3 \times 10^{-2}$ & 1.6 & $4.0 \times 10^{-3}$ & $2.7 \times 10^{-2}$ \\
& 3 & $4.9 \times 10^{-4}$ & $3.4 \times 10^{-3}$ & $8.7 \times 10^{-5}$ & $5.9 \times 10^{-4}$ \\
& 4 & $1.1 \times 10^{-5}$ & $7.3 \times 10^{-5}$ & $1.9 \times 10^{-6}$ & $1.3 \times 10^{-5}$ \\
\hline \multirow{4}{*}{300} & 1 & 3.9 & - & 1.5 & 5.1 \\
& 2 & $2.0 \times 10^{-1}$ & 1.3 & $6.7 \times 10^{-2}$ & $2.4 \times 10^{-1}$ \\
& 3 & $9.1 \times 10^{-3}$ & $5.6 \times 10^{-2}$ & $3.1 \times 10^{-3}$ & $1.1 \times 10^{-2}$ \\
& 4 & $4.2 \times 10^{-4}$ & $2.6 \times 10^{-3}$ & $1.4 \times 10^{-4}$ & $5.1 \times 10^{-4}$ \\
& 5 & $1.9 \times 10^{-5}$ & $1.2 \times 10^{-4}$ & $6.4 \times 10^{-6}$ & $2.3 \times 10^{-5}$ \\
\hline
\end{tabular}

(b) $\tau_{1}=10^{\circ}, \tau_{2}=-10^{\circ}$

\begin{tabular}{cccccc}
\hline$x_{P}[\mathrm{~m}]$ & $n$ & $\Delta y_{\mathrm{Q} n 1}[\mathrm{~m}]$ & $\Delta y_{\mathrm{Q} n 2}[\mathrm{~m}]$ & $\Delta \alpha_{1}[\mathrm{deg}]$ & $\Delta \alpha_{2}[\mathrm{deg}]$ \\
\hline & 1 & 5.6 & - & 3.3 & 9.4 \\
& 2 & $7.5 \times 10^{-1}$ & 2.4 & $3.8 \times 10^{-1}$ & 1.1 \\
50 & 3 & $8.8 \times 10^{-2}$ & $2.6 \times 10^{-1}$ & $4.4 \times 10^{-2}$ & $1.3 \times 10^{-1}$ \\
& 4 & $1.0 \times 10^{-2}$ & $3.0 \times 10^{-2}$ & $5.1 \times 10^{-3}$ & $1.5 \times 10^{-2}$ \\
& 5 & $1.2 \times 10^{-3}$ & $3.5 \times 10^{-3}$ & $5.8 \times 10^{-4}$ & $1.7 \times 10^{-3}$ \\
& 6 & $1.4 \times 10^{-4}$ & $4.0 \times 10^{-4}$ & $6.8 \times 10^{-5}$ & $2.0 \times 10^{-4}$ \\
& 7 & $1.6 \times 10^{-5}$ & $4.6 \times 10^{-5}$ & $7.8 \times 10^{-6}$ & $2.3 \times 10^{-5}$ \\
\hline & 1 & 5.8 & - & 7.8 & 11.6 \\
300 & 2 & 1.6 & 5.4 & 1.9 & 2.8 \\
& 3 & $3.9 \times 10^{-1}$ & 1.2 & $4.4 \times 10^{-1}$ & $6.8 \times 10^{-1}$ \\
& 4 & $9.3 \times 10^{-2}$ & $2.7 \times 10^{-1}$ & $1.1 \times 10^{-1}$ & $1.6 \times 10^{-1}$ \\
& 5 & $2.2 \times 10^{-2}$ & $6.4 \times 10^{-2}$ & $2.5 \times 10^{-2}$ & $3.9 \times 10^{-2}$ \\
& 6 & $5.3 \times 10^{-3}$ & $1.5 \times 10^{-2}$ & $6.0 \times 10^{-3}$ & $9.3 \times 10^{-3}$ \\
& 7 & $1.3 \times 10^{-3}$ & $3.6 \times 10^{-3}$ & $1.4 \times 10^{-3}$ & $2.2 \times 10^{-3}$ \\
& 8 & $3.0 \times 10^{-4}$ & $8.7 \times 10^{-4}$ & $3.4 \times 10^{-4}$ & $5.3 \times 10^{-4}$ \\
& 9 & $7.2 \times 10^{-5}$ & $2.1 \times 10^{-4}$ & $8.2 \times 10^{-5}$ & $1.3 \times 10^{-4}$ \\
\hline
\end{tabular}

Figures 4-6 show the change of the total electric field $\left|\mathbf{E}^{t}\right|=E^{t}$ and its incident and diffracted components with a distance $x_{P}$ from a surface of the wedge 2 ; when the wave is incident to the perfectly conducting wedges whose inner angles are $\theta_{i 0}=\theta_{\text {in }}=0^{\circ}(i=1,2)$, the heights of source and two edges are $h_{S}=40 \mathrm{~m}, h_{1}=10 \mathrm{~m}$, and $h_{2}=30 \mathrm{~m}$.

Figure 4 shows the frequency characteristics of the fields when the horizontal polarized wave $(\widehat{\mathbf{i}}=\widehat{\mathbf{y}})$ is incident and $\phi_{1}=\phi_{2}=\tau_{1}=\tau_{2}=0^{\circ}$. In this figure, the fields are the total fields $\left(E_{y}^{t}\right)$, single diffracted fields $\left(E_{2, y}^{d 1}\right)$ by the $e_{2}$, double diffracted components $\left(E_{(1), y}^{d 2}\right)$, and the slope diffracted components $\left(E_{(2), y}^{d 2}\right)$, where the subscript $y$ means $y$ component. $E^{S P}$ is the direct field from $S$ to $P$ without wedges. At $x_{P}=285 \mathrm{~m}$ the direct field $\mathbf{E}^{\mathrm{inc}}$ appears, so we called the point $x_{P}=285.0 \mathrm{~m} \mathrm{SB}_{i}$ (shadow boundary for an incident wave) and the region near to $x_{P}=285 \mathrm{~m}$ is called a transition region. When the frequency is doubled, outside the transition

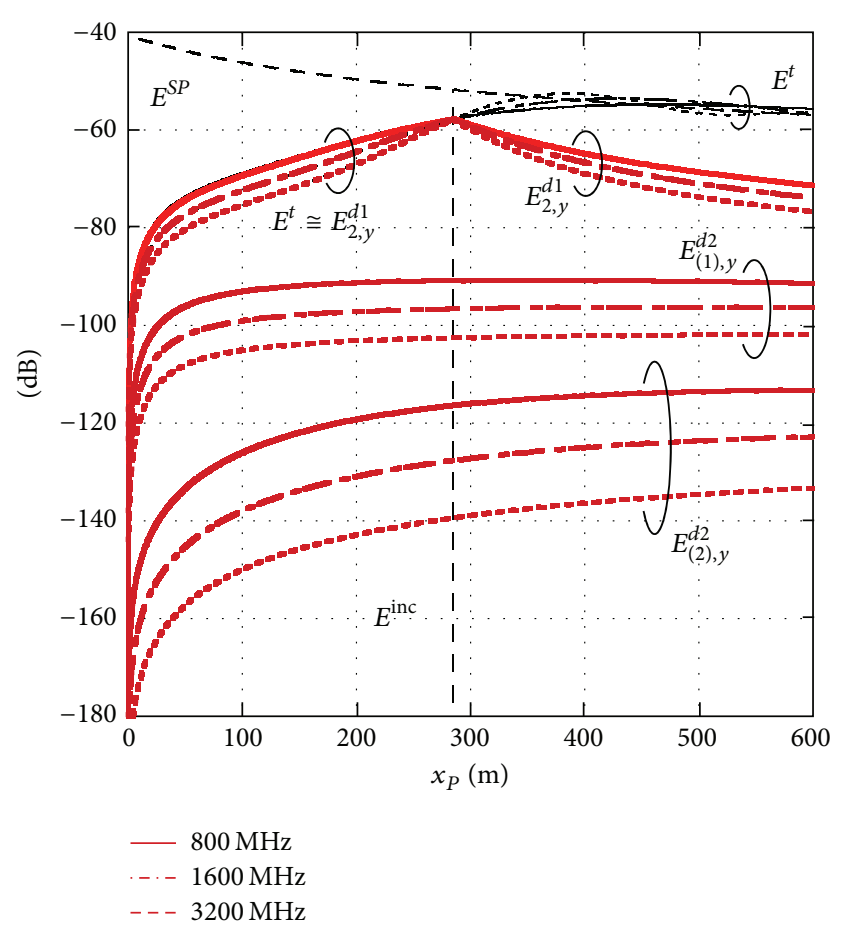

FIGURE 4: Fields diffracted by two wedges and their components for three frequencies $(800,1600$, and $3200 \mathrm{MHz})\left(\widehat{\mathbf{i}}=\widehat{\mathbf{y}}, h_{1}=h_{2}=30 \mathrm{~m}\right.$, and $\psi_{1}=\psi_{2}=\tau_{1}=\tau_{2}=0^{\circ}$ ).

region, $E_{2, y}^{d, 1}, E_{(1), y}^{d, 2}$, and $E_{(2), y}^{d, 2}$ are deceased at $3 \mathrm{~dB}, 6 \mathrm{~dB}$, and $12 \mathrm{~dB}$, respectively [13]. For example, at $x_{P}=100 \mathrm{~m} E_{2, y}^{d, 1}$ is $-69.3 \mathrm{~dB},-72.4 \mathrm{~dB}$, and $-75.4 \mathrm{~dB}$ at $800 \mathrm{MHz}, 1600 \mathrm{MHz}$, and $3200 \mathrm{MHz}$, and $E_{(1), y}^{d, 2}$ and $E_{(2), y}^{d, 2}$ are attenuated at $6 \mathrm{~dB}$ and $12 \mathrm{~dB}$ when frequency is doubled.

Figure 5 shows the fields when the horizontal polarized wave $(\widehat{\mathbf{i}}=\widehat{\mathbf{y}})$ is incident on the wedges. Figure $5(\mathrm{a})$ is the case when two edges $e_{1}$ and $e_{2}$ are parallel $\left(\psi_{1}=\psi_{2}=\tau_{1}=\tau_{2}=\right.$ $\left.0^{\circ}\right)$. Only horizontal wave $E_{y}$ is diffracted and the total field $E^{t}\left(=\left|E_{y}^{i}+E_{y}^{d 1}+E_{(1), y}^{d 2}+E_{(2), y}^{d 2}\right|\right)$ increases according to distance $x_{P}$ because in this case the direct field $E^{\text {inc }}$ is added in the range $x_{P} \geq 285.0 \mathrm{~m}$.

Figure 5(b) is the case when $\psi_{1}=30^{\circ}, \psi_{2}=-30^{\circ}$, and $\tau_{1}=\tau_{2}=0$. As two edges are rotated in horizontal plane, the orthogonal components $\left(E_{x}, E_{z}\right)$ are generated, and when the angle between two edges increases, relatively strong orthogonal polarized wave components appear to the incident wave. But the horizontal components $\left(E_{y}^{t}, E_{2, y}^{d 1}\right.$, $E_{(1), y}^{d 2}$, and $\left.E_{(2), y}^{d 2}\right)$ change scarcely from Figure 5(a).

Figure 5(c) shows the case that two edges are not rotated in horizontal plane but in vertical plane $\left(\psi_{1}=\psi_{2}=0^{\circ}\right.$, $\tau_{1}=30^{\circ}$, and $\left.\tau_{2}=-30^{\circ}\right)$. Comparing Figure 5(c) with Figure 5(b), the orthogonal $(z, x)$ components are different from each other, but the principal components $\left(E^{t}, E_{y}^{i}\right.$, and $\left.E_{2, y}^{d 1}\right)$ are almost the same as in Figures 5(a) and 5(b).

Figure 6 shows fields for vertically polarized incidence $(\widehat{\mathbf{i}}=\widehat{\mathbf{z}})$. There are fields diffracted in $x$ and $z$ components. 


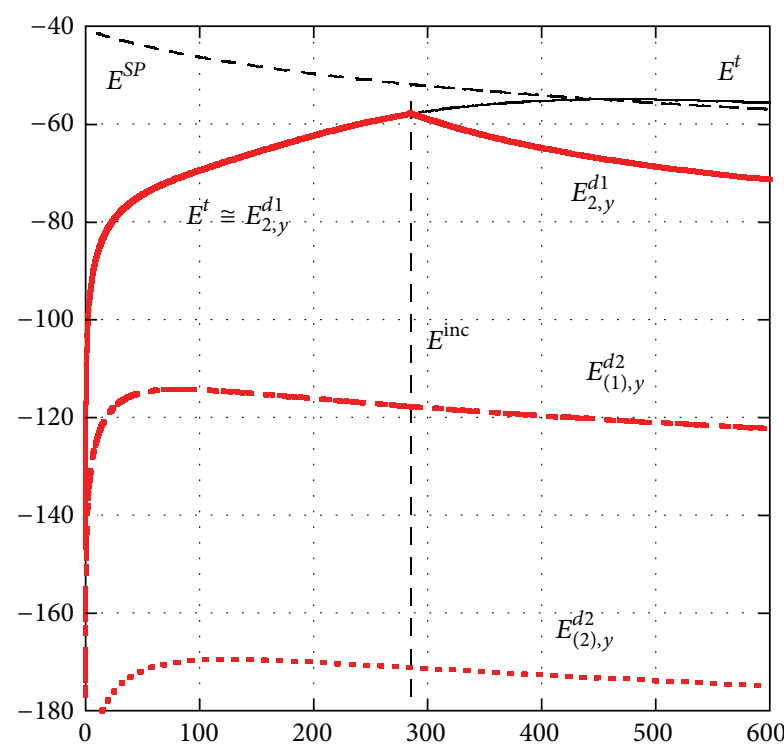

(a) $\psi_{1}=\psi_{2}=\tau_{1}=\tau_{2}=0^{\circ}$

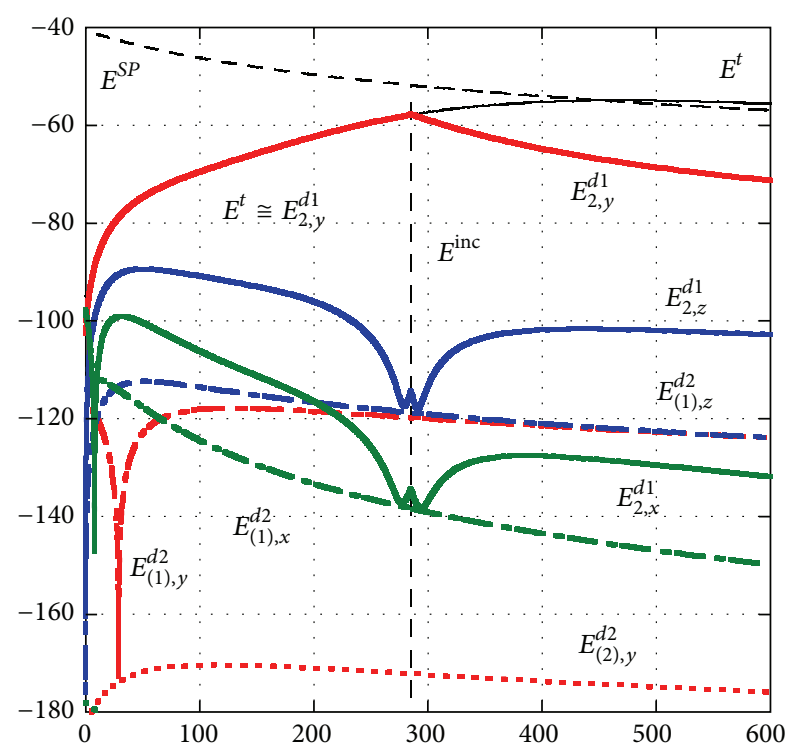

(b) $\psi_{1}=30^{\circ}, \psi_{2}=-30^{\circ}, \tau_{1}=\tau_{2}=0^{\circ}$

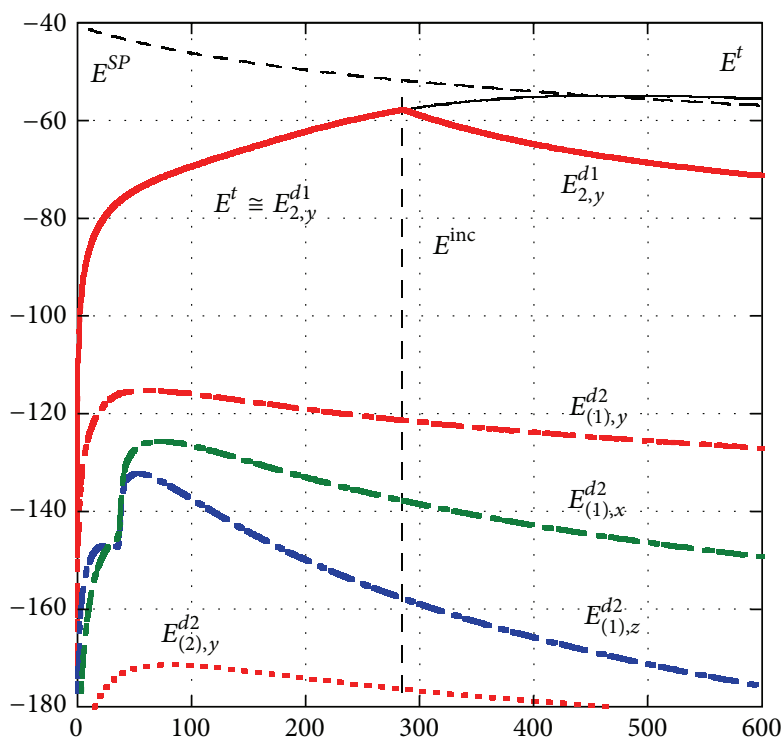

(c) $\psi_{1}=\psi_{2}=0^{\circ}, \tau_{1}=30^{\circ}, \tau_{2}=-30^{\circ}$

FIGURE 5: Fields diffracted by two wedges for horizontal polarized incidence.

So, the total field $E^{t}$ is composed by $x$ and $z$ components. In Figure 6(a) the case when the edges $e_{1}$ and $e_{2}$ are parallel $\left(\psi_{1}=\psi_{2}=\tau_{1}=\tau_{2}=0^{\circ}\right)$ is shown. Comparing Figure 6(a) with Figure 5(a), in Figure 6(a), the $x$ components of the diffracted fields $E_{2, x}^{d 1}, E_{(1), x}^{d 2}$, and $E_{(2), x}^{d 2}$ at $x_{P}=0$ are not zero because of the boundary condition for conductor. Then, the curve of $E^{t}$ starts with finite value at $x_{P}=0$, but in Figure 5(a) the $E^{t}$ is zero. But both $E^{t}$ curves in Figures 5(a) and 6(a) almost agree in the region where $x_{P}$ is large. Figure $6(\mathrm{~b})$ is the fields when $\psi_{1}=30^{\circ}, \psi_{2}=-30^{\circ}$, and $\tau_{1}=\tau_{2}=0^{\circ}$. The orthogonal components $E_{2, y}^{d 1}, E_{(1), y}^{d 2}$, and $E_{(2), y}^{d 2}$ are generated, but the principal components $\left(E^{t}, E^{i}, E_{2, z}^{d 1}\right.$, and $\left.E_{2, x}^{d 1}\right)$ change a little bit. Figure $6(\mathrm{c})$ shows the case for $\psi_{1}=\psi_{2}=0^{\circ}, \tau_{1}=30^{\circ}$, and $\tau_{2}=-30^{\circ}$. The $y$ component of single diffracted wave $E_{2, y}^{d 1}$ is zero, and the double diffracted components $\left(E_{(1), z}^{d 2}, E_{(1), x}^{d 2}\right.$, etc.) are different from those of Figure 6(b), but the principal components are almost the same as in Figures 6(a) and 6(b).

\section{Conclusions}

In this paper, we considered the diffracted fields by two edges, parallel edges and edges with arbitrary angle. We used three coordinate systems \#0, \#1, and \#2 and considered coordinate transformation. Next, we explained the method to find the diffraction points on two edges. We analyzed the diffracted fields by two edges using the GTD. The numerical 


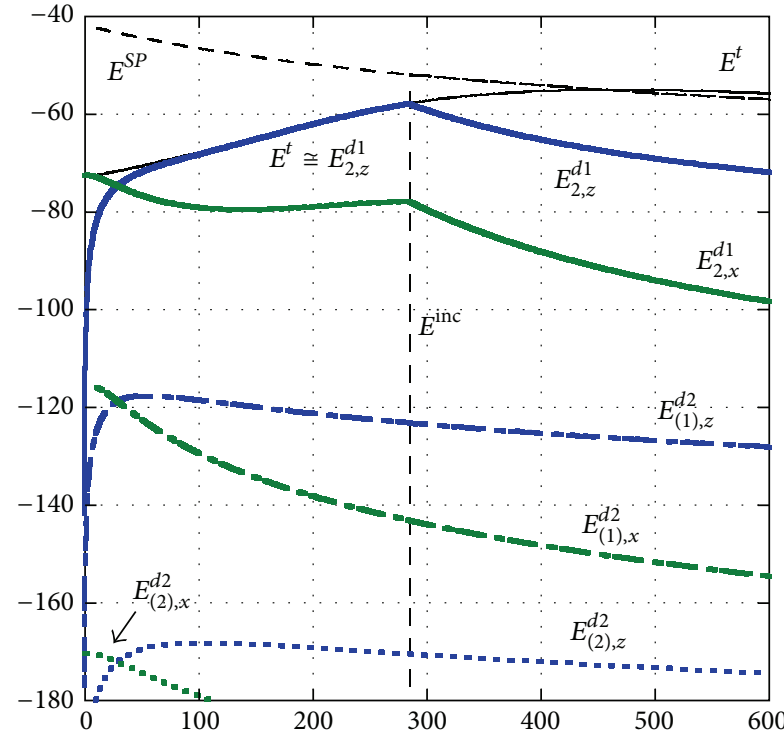

(a) $\psi_{1}=\psi_{2}=\tau_{1}=\tau_{2}=0^{\circ}$

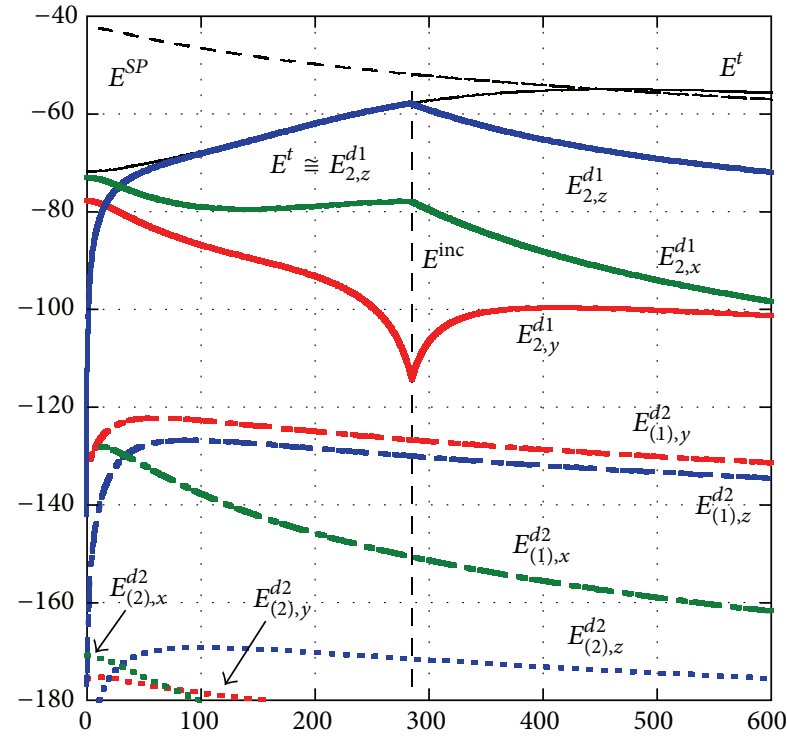

(b) $\psi_{1}=30^{\circ}, \psi_{2}=-30^{\circ}$, and $\tau_{1}=\tau_{2}=0^{\circ}$

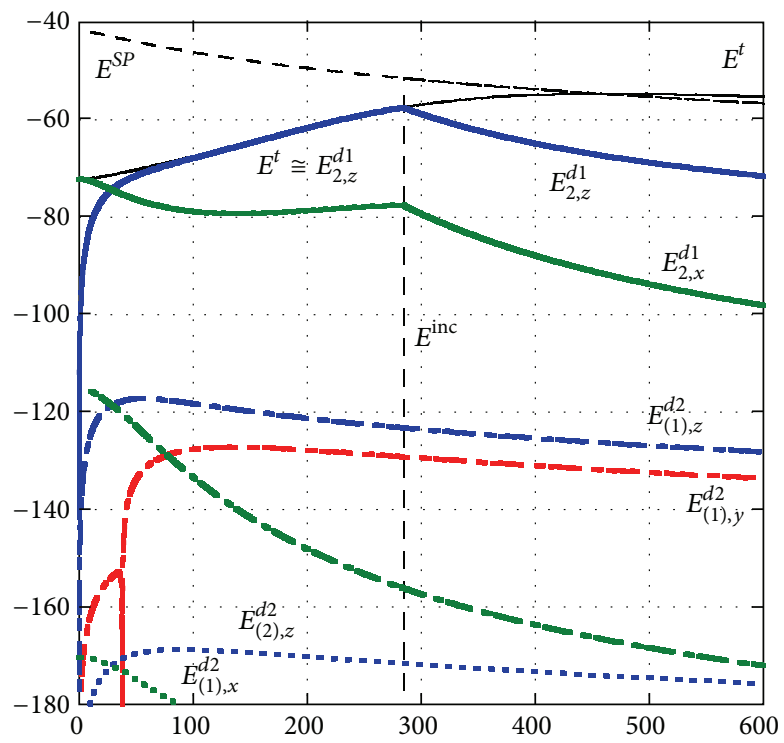

(c) $\psi_{1}=\psi_{2}=0^{\circ}, \tau_{1}=30^{\circ}$, and $\tau_{2}=-30^{\circ}$

FIGURE 6: Fields diffracted by two wedges for vertical polarized incidence.

calculations were carried out for two wedges of perfect conductor for horizontal and vertical polarized incidence.

From the numerical results, we conclude as follows.

(i) The diffraction points on two edges with arbitrary angle in horizontal and vertical planes can be confirmed by few repetitions of our method.

(ii) The number of repetitions is increased when the angles and/or distance to the observation point are increased.

(iii) When the frequency is doubled, outside the transition region, the single-, double-, and slope-diffracted fields are deceased $3 \mathrm{~dB}, 6 \mathrm{~dB}$, and $12 \mathrm{~dB}$, respectively. (iv) When the wave is incident perpendicularly to parallel edges, the diffracted fields have the same component as incident polarization, but the orthogonal component wave to incident wave is generated by edges with arbitrary angle.

(v) When the angle between two edges increases horizontally and/or vertically, relatively strong orthogonal polarized wave components appear, but the principal components are the same as the incident wave.

In the future work, we would like to confirm numerical results by experiments. 


\section{Conflict of Interests}

The authors declare that there is no conflict of interests regarding the publication of this paper.

\section{References}

[1] A. J. Goldsmith and L. J. Greenstein, "A measurement-based model for predicting coverage areas of urban microcells," IEEE Journal on Selected Areas in Communications, vol. 11, no. 7, pp. 1013-1023, 1993.

[2] T. Imai and T. Fujii, "A propagation prediction system for urban area using ray-tracing method," Technical Report of IEICE RCS97-37, 1997 (Japanese).

[3] W. Zhang, "A wide-band propagation model based on utd for cellular mobile radio communications," IEEE Transactions on Antennas and Propagation, vol. 45, no. 11, pp. 1669-1678, 1997.

[4] J. Iwashige, L. Barolli, Y. Fukuyama, and H. Iwami, "Electromagnetic fields diffracted by two edges with arbitrary angle in a plane," International Journal of Microwave and Optical Technology, vol. 1, no. 2, pp. 854-859, 2006.

[5] J. Iwashige and L. Barolli, "Electromagnetic fields diffracted by two horizontal edges with arbitrary angle for different and same heights," International Journal of Computer Systems Science and Engineering, vol. 25, no. 2, pp. 1-8, 2010.

[6] K.H.Ng, E. K. Tameh, and A. R. Nix, "A new heuristic geometrical approach for finding non-coplanar multiple edge diffraction ray paths," IEEE Transactions on Antennas and Propagation, vol. 54, no. 9, pp. 2669-2672, 2006.

[7] M. Iwaida, J. Iwashige, S. Kameyama, and R. Nagao, "A method for finding diffraction points on two edges with arbitrary angle and its diffracted fields," in Proceedings of the 7th International Conference on Complex, Intelligent, and Software Intensive Systems (CISIS '13), pp. 207-212, July 2013.

[8] S. Kameyama, J. Iwashige, M. Iwaida, and Y. Isayama, "Diffracted fields by the edge of wedge and reflected fields from surface of wedge," in Proceedings of the 7th International Conference on Complex, Intelligent, and Software Intensive Systems (CISIS '13), pp. 201-206, July 2013.

[9] J. B. Keller, "Geometrical theory of diffraction," Journal of the Optical Society of America, vol. 52, no. 2, pp. 116-130, 1962.

[10] R. G. Kouyoumjian and P. H. Pathak, "A uniform geometrical theory of diffraction for an edge in perfectly conducting surface," Proceedings of the IEEE, vol. 62, no. 11, pp. 1448-1461, 1974.

[11] P. D. Holm, "UTD-diffraction coefficients for higher order wedge diffracted fields," IEEE Transactions on Antennas and Propagation, vol. 44, no. 6, pp. 879-888, 1996.

[12] P. D. Holm, "A new heuristic UTD diffraction coefficient for nonperfectly conducting wedges," IEEE Transactions on Antennas and Propagation, vol. 48, no. 8, pp. 1211-1219, 2000.

[13] J. Iwashige, L. Barolli, and K. Hayashi, "Frequency characteristics of diffracted waves going over building rooftop," in Proceedings of the 5th International Conference on Complex, Intelligent and Software Intensive Systems (CISIS '11), pp. 442447, July 2011. 

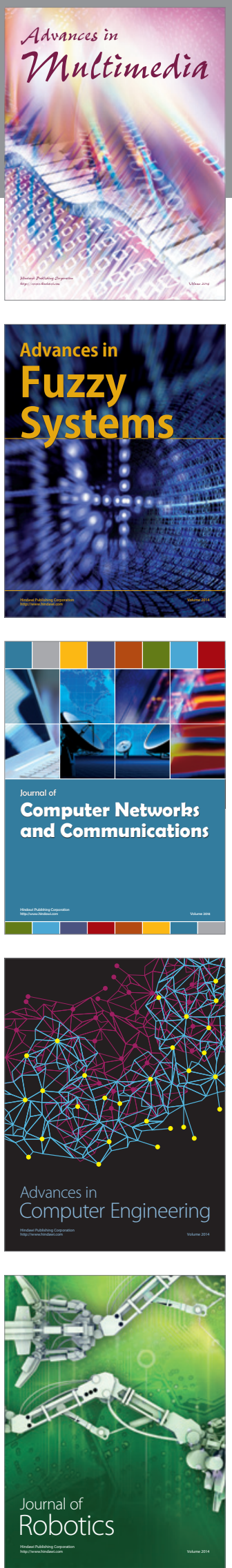

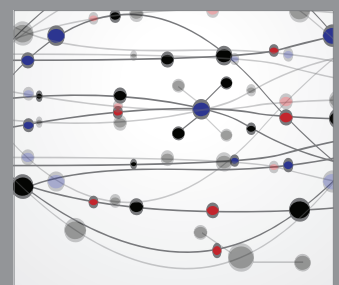

The Scientific World Journal
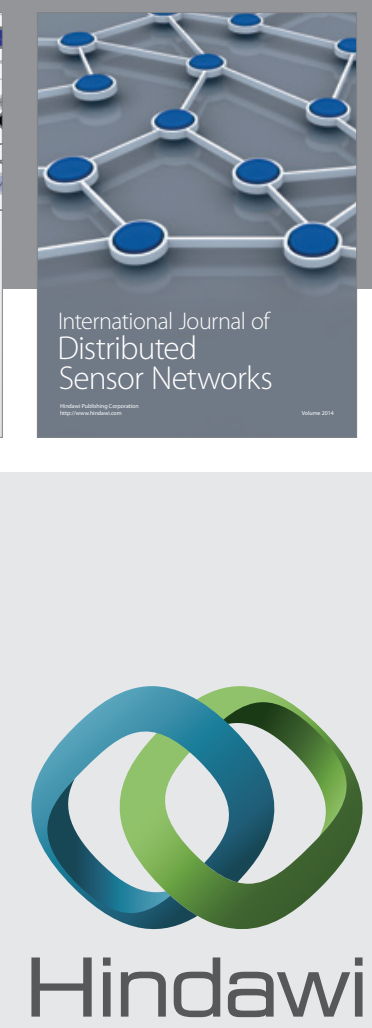

Submit your manuscripts at

http://www.hindawi.com
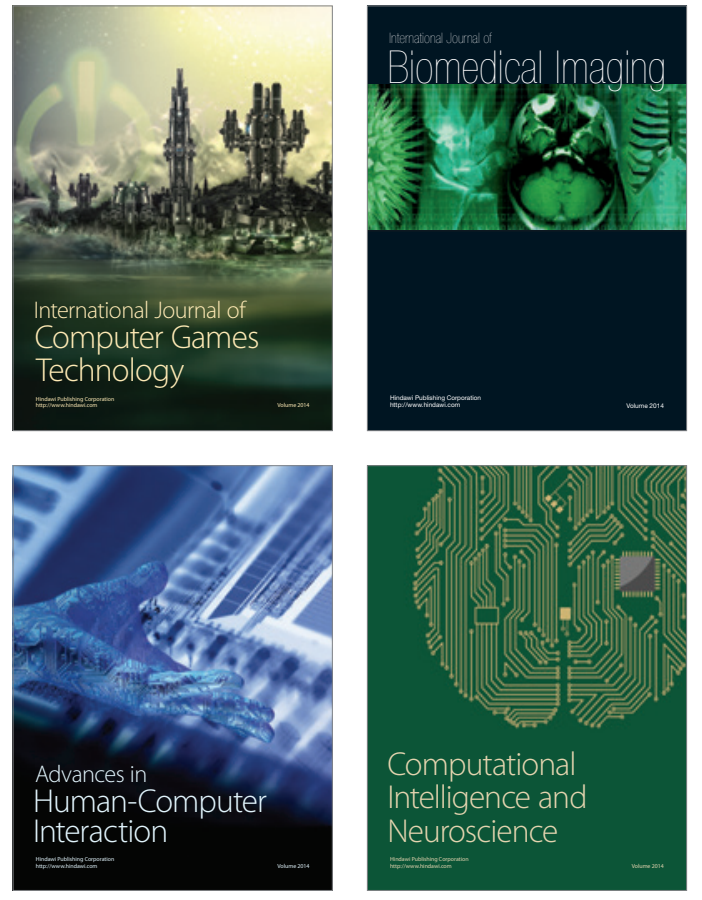
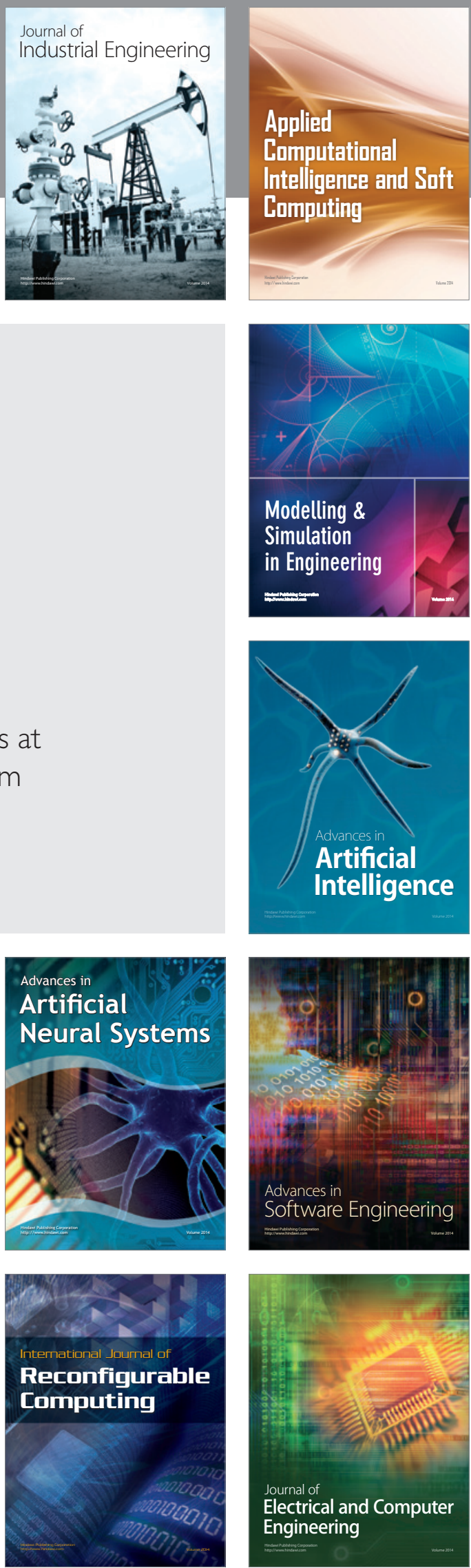